Cinémas

Revue d'études cinématographiques

Journal of Film Studies

\title{
ROPARS-WUILLEUMIER, Marie-Claire. Écraniques. Le film du texte. Lille : Presses universitaires de Lille, 1990, 228 p.
}

\section{Lucie Roy}

Volume 3, numéro 1, automne 1992

URI : https://id.erudit.org/iderudit/1001185ar

DOI : https://doi.org/10.7202/1001185ar

Aller au sommaire du numéro

Éditeur(s)

Cinémas

ISSN

1181-6945 (imprimé)

1705-6500 (numérique)

Découvrir la revue

Citer ce compte rendu

Roy, L. (1992). Compte rendu de [ROPARS-WUILLEUMIER, Marie-Claire.

Écraniques. Le film du texte. Lille : Presses universitaires de Lille, 1990, 228 p.]

Cinémas, 3(1), 149-153. https://doi.org/10.7202/1001185ar d'utilisation que vous pouvez consulter en ligne.

https://apropos.erudit.org/fr/usagers/politique-dutilisation/ 
ROPARS-WUILLEUMIER, Marie-Claire. Écraniques. Le film du texte. Lille : Presses universitaires de Lille, 1990, 228p.

“Nous ne lisons pas sans rêver; et si parfois nous analysons, e'est pour renouveler le plaisir d'errer."

Ropars

Cette œuvre de Marie-Claire Ropars-Wuilleumier, Écraniques, colossale par son contenu, rassemble des textes déjà publiés, d'autres inédits et vient notamment à la suite de son ouvrage : Le Texte divisé. Ce dernier examinait l'écriture filmique et la loi intervallique du montage et cherchait, ce faisant, à faire état de la circularité du sens et de l'énonciation filmiques.

Bien que l'auteure souligne à plusieurs reprises l'aspect «essayiste» de l'actuelle entreprise ou, mieux, celui de la prise et de la saisie de l'écriture de la modernité, Écraniques marque un pas décisif quant à l'enquête portant sur l'errance discursive de cette écriture, même filmique, que l'analyse du texte par le film suffit à réfléchir : «(...) la modernité rend à l'œuvre l'opacité formelle d'un acte qui ne sera dit de langage que parce qu'il agit par et contre le seul langage (...)» (p. 29).

Traversant le texte du film, le film, lecteur du texte, voire le film et le texte absents appelés dans la textualité, l'exploration de l'écriture par le film fait théoriquement appel au film comme opérateur des différences entre la voix et le silence, entre l'image esquissée et arrêtée dans le mouvement de «(...) la transitivité du discours» (p. 29) par l'énonciation même de son absence dans la chute du texte. L'auteure fait, en d'autres termes, référence à la notion de montage filmique comme s'il s'agissait d'une sorte de lieu spéculaire de l'écriture, «l'expérience porte [donc] moins sur les matériaux que sur les opérations (...)» (p. 25). Lesquels matériaux sont, à l'évidence, représentatifs d'une certaine hétérogénéité initiale, laquelle, comme on le sait, désigne le fait de mobilisation des images, des musiques, des bruits, et des voix et appelle, par conséquent, une sorte d'hétérogénéité autre, celle du «hors» et du of découpés en et par lui. Or, cette hétérogénéité sert précisément le «démontage» du texte littéraire qui, illusoirement homogène sur le plan linguistique, retient d'une certaine façon les espaces off de l'écriture et appelle, en ce sens, d'autres lieux de la signification.

Hors-champ du texte appelé dans le champ du texte, découpe, contrariété, annulation, déroute, dévoiement plutôt qu'«instrumentalité des signes», l'auteure emprunte, autrement, la 
course des réflexions de Blanchot concerriant la parole en interrogeant le paradoxal état de l'écriture qui retient la réécriture dans l'écriture du texte. L'analyse tend ainsi à laisser entendre le non-écrit de l'écriture, à prendre en compte l'ébranlement du texte, la rupture, la discontinuité, comme moment irradiant de la parole et «(...) respiration du discours» (Blanchot, p. 108).

Cette œuvre se fonde, en clair et tel que l'auteure le souligne, sur le principe d'une «analecture» qui laisse présager du doute d'une «(...) pensée de l'impensable (...)» (p. 225) et, on l'a suffisamment noté, elle se fonde sur la pratique d'une «filmolecture» d'œuvres d'écrivains et/ou de cinéastes tels que Duras/Resnais, Klossowski/Ruiz, Aquin/Aquin, Des Forêts/Des Forêts, Mann/Visconti.

Double et disjonctif, le film multiplie, dans le texte, le circuit de l'errance; mais rigoureux, il nous contraint à délimiter strictement les voies de l'hétérogène, par où se perpétue le paradoxe du texte : un paradoxe n'a pas à être résolu, mais au contraire exploré - telle est du moins la règle du texte, que l'emprunt filmique permet d'accroître (p. 227).

Miroir théorique des œuvres de la modernité, l'ouvrage emprunte volontairement la «forme mouvante» d'un engendrement analytique du paradoxe où, par définition, une fois posé le terme initial, l'auteure examine son contraire, le signe enferme à la fois son absence et son ouverture sur d'autres signes, l'énoncé retient encore l'indicible de l'écrit. Marie-Claire Ropars s'attache donc d'abord à établir les visées et les précautions théoriques de l'analyse et ouvre son propos sur la traversée du texte dans le film par l'étude de «l'atomisation», des égarements formels et narratifs du film de Duras et Resnais, Hiroshima mon amour, et par celle de l'opération de démontage textuel de l'œuvre de Blanchot, L'Arrêt de mort.

Ce film de Duras et Resnais donne, comme on le sait, à lire des images de la guerre et fait, en quelque sorte, taire l'image et la voix, celle principalement de la narratrice qui, par opposition ou diffraction, s'étiole aux arêtes des images. L'analyse de ce film met donc en évidence la disjonction - et donc la possible jonction - de la bande image par rapport à la bande son et sert, ce faisant, à éclairer la présence d'un off qui «(...) double négativement l'énoncé (...)» (p. 54) et permet, à ce propos, de mieux comprendre le travail narratif du texte dans le film :

(...) en mettant en scène le translert opéré par la jeune femme de son amour allemand sur un amant japonais, le film masquera le transfert qu'il propose au spectateur de l'innommable d'Hiroshima - côté de l'écriture - sur le «racontable» de Nevers - côté de la parole; 
détournant ainsi l'écriture vers le récit après avoir changé l'Histoire en écriture (pp. 53-54).

Examinant le tracement ou le montage de l'impossible récit dans le roman de Blanchot, L'Arrêt de mort, Ropars démontre, de plus, comment par glissements et versions contradictoires, le livre s'arrache du récit et davantage, comment :

(...) plus le texte se développe, plus il assure sa propre béance : [par des] failles de raccords [et des] passages au noir de plus en plus étendus, [par la] multiplication des échos et des reflets internes, qui déjouent la délimitation des paragraphes; et, pour finir, [par] l'interruption, où vacille la temporalité (pp. 53-54).

Euvre plurielle par rapport aux incessants débordements des textes dans le texte et par rapport au dérobement du texte par des personnages/narrateurs éclatés, le film La Femme du Gange et le livre Le Ravissement de Lol $V$. Stein de Duras font ici état d'une déperdition de la narration au profit d'une écriture par là exhibée dans le sinueux parcours du texte. Ropars se penche entre autres ici sur l'usage du plus-que-parfait dans le texte littéraire, sur le passage du je au $i l$, sur la perte d'identité des personnages menacés par une sorte d'oubli et par l'absence d'un off qui, même informulé, les retient aux abords du texte, sur la menace d'un nonvoir appelé par le voir, tous ces éléments contribuant à fragmenter et à dédoubler le récit littéraire et le récit filmique. Mis en texte par une narration déformée et tronquée, le texte de Duras se tient dans l'intervalle et la disjonction et retient le travail de l'écriture dans le tissu du texte par lequel le récit, ébranlé, ne saurait être apaisé. Il s'agit d'un récit, mais gangrené par l'immensité de l'imprononcé et le doute d'un texte hors-texte rappelé dans le site même du texte.

Les textes de la modernité ne souffrent pas, tel que le démontre ici Ropars, uniquement de l'appel des voix et des récits qui, dans le hors-champ du texte se trouvent rappelés dans le texte, mais ils souffrent également de la distance inaugurée par le dire en rapport avec le langage. L'analyse laisse croire que, chez Klossowski, si la représentation est en fuite c'est, dit sommairement, sans doute pour rompre le maléfice qui veut que l'énoncé ne peut témoigner de l'entièreté de la pensée. L'auteur, Klossowski, se voit ainsi forcé d'innocenter le dire en le tenant dans le non-vrai, le non-faux, le..., et de rappeler dans le texte la dispersion même du sens comme visée énonciative et ce, notamment, par l'exclusion d'un je qui, comme la parole, demeure irreprésentable ou trompé par son propre discours. Dans le film de Ruiz, lecteur du texte de Klossowski, La Vocation suspendue, Ropars montre comment 
s'opère ce travail de démantèlement. «Les signes affluent, à la fois vus et entendus, disjoints et non séparables (...)» (p.93). Le film sert, ici encore, de révélateur dans la mesure où il joue le jeu d'un dédoublement formel et d'un double ratage : les séquences, à titre d'exemple, des deux hypothétiques films dans le film demeurent, semble-t-il, «(...) non sécables en parties différentes (...)» (p. 94) de sorte que le lecteur n'a pour ainsi dire pas de prise sur le texte. Faute, encore une fois, d'assumer le dire - en assurant dans le film le lire plutôt que le dire - , le je comme le $t u$ sont comme mis en abysse d'un on anonyme : l'auteur et le lecteur se trouvent, ce faisant, détroussés, désavoués, véritablement exclus :

\begin{abstract}
Plus que toute autre ceuvre de Klossowski, La vocation suspendue permet ainsi de dénouer la chaîne des opérations qui de lecture barrée en lecteur simulé, et d'énonciation dérobée en énoncé dénoncé, tracent le cercle d'une écriture où le langage se dit dans le dédire de la logique (p. 110).
\end{abstract}

Partant du champ de la parole et, plus particulièrement, de l'étude de la voix off, Ropars étend le paradoxe de la voix, de ses références et, davantage, de ses inférences, dans les frontières du texte littéraire de Des Forêts, La Mémoire démentielle. Rappelant le «film absent dans le texte», cet ouvrage fait de plusieurs façons appel au montage : montage vertical, l'écrit retient le taire de la voix; montage horizontal, le récit, séquentiellement annoté, se trouve paré de blancs, d'intervalles. Or, il faut bien voir que l'analyse même de la voix off, particulière au cinéma, a fait en sorte d'offrir à la littérature une sorte de miroir — ou de mémoire — : «(...) entre lecteur et lecture, l'image multipliée de l'écriture, par qui seule nous lisons» (p. 26), reflétant ainsi la fuite de l'écrire dans l'écriture. Et encore :

Ainsi le modèle filmique qui informe obliquement la mise en mémoire du récit véhicule-t-il moins la plénitude des représentations qu'il ne contraint à suivre le tracé d'un double montage en disjonction, où l'espace sonore, par récession ou par excès, accroît l'opacité d'une chaîne visuelle faite de morceaux rapportés (p. 143).

Pour conclure ce compte rendu qui, à l'évidence, ne saurait témoigner de l'entièreté des propositions théoriques avancées, des nuances qui y sont apportées et, même, des œuvres qui y sont abordées - les analyses des œuvres d'Aquin, de Des Forêts et, enfin, du couple Mann/Visconti n'ont que très peu ou pas été évoquées -, on aimerait citer longuement les deux dernières phrases de la conclusion de cet ouvrage, Écraniques, qui loin de fermer le débat mettent en évidence la nécessité d'une perpétuelle relance de l'entreprise : 
Active, instable, l'éviction de la personne, si remarquable en Klossowski ou Blanchot déploie le libre champ d'une subjectivité que n'accapare aucun sujet; mais pour rendre audible la voix souterraine qui traverse l'écriture de ces cuvres déjà lointaines, il a fallu passer par l'explosion souveraine de cinéastes qui, longtemps après, Resnais ou Ruiz, mettent en pleine lumière la sédition de textes capables à la fois de séduire et de se séparer. Nécessité du cinéma, que déclare Duras lorsqu'elle se fait le destructeur de l'identité textuelle en devenant scripteur de film; dérivation filmique érigée en principe par Visconti l'échangeur de récits; ou ruse d'une analogie cinématographique que Des Forêts et Aquin simulent en se l'appropriant : en tous les cas le désastre du livre va de pair avec la dissimulation de l'œuvre. À cette attaque du texte peut seule répondre une lecture qui ne serait ni jeu de rôles ni jouissance complice, mais marche frontalière, esquive et attirance : une lecture jalouse, peut-être (p. 227).

Lucie Roy

Université Laval

OUVRAGE CITÉ

Blanchot, Maurice. L'Entretien infini. Paris : Gallimard,1983. 